\title{
Papers
}

\section{Association between competing interests and authors' conclusions: epidemiological study of randomised clinical trials published in the $B M J$}

Lise L Kjaergard, Bodil Als-Nielsen

\begin{abstract}
Objective To assess the association between competing interests and authors' conclusions in randomised clinical trials.

Design Epidemiological study of randomised clinical trials published in the $B M J$ from January 1997 to June 2001. Financial competing interests were defined as funding by for profit organisations and other competing interests as personal, academic, or political. Studies 159 trials from 12 medical specialties. Main outcome measures Authors' conclusions defined as interpretation of extent to which overall results favoured experimental intervention.

Conclusions appraised on 6 point scale; higher scores favour experimental intervention.

Results Authors' conclusions were significantly more positive towards the experimental intervention in trials funded by for profit organisations alone compared with trials without competing interests (mean difference 0.48 (SE 0.13), $\mathrm{P}=0.014$ ), trials funded by both for profit and non-profit organisations (0.30 (SE 0.10), $\mathrm{P}=0.003)$, and trials with other competing interests (0.45 (SE 0.13), $\mathrm{P}=0.006)$. Other competing interests and funding from both for profit and non-profit organisations were not significantly associated with authors' conclusions. The association between financial competing interests and authors' conclusions was not explained by methodological quality, statistical power, type of experimental intervention (pharmacological or non-pharmacological), type of control intervention (for example, placebo or active drug), or medical specialty.

Conclusions Authors' conclusions in randomised clinical trials significantly favoured experimental interventions if financial competing interests were declared. Other competing interests were not significantly associated with authors' conclusions.
\end{abstract}

\section{Introduction}

Financial and other competing interests have recently received increasing attention. ${ }^{1}$ In a study of trials of multiple myeloma the authors' conclusions-that is, the authors' reported interpretation of the overall trial results-were more positive towards the benefit of experimental interventions in those trials that were funded by the pharmaceutical industry compared with trials that were funded by non-profit organisations. ${ }^{2}$ In a systematic review on antipsychotic drugs for schizophrenia, trials were more likely to show a benefit of treatment if they were funded by drug companies. ${ }^{3}$ It is not known whether the association between financial competing interests and authors' conclusions is limited to certain specialties or whether it is a general problem. It is also not known whether personal, academic, or political interests are associated with authors' conclusions.

Unlike most journals, the $B M J$ requires authors to declare funding as well as other competing interests. Trials in the $B M J$ therefore offer a unique opportunity to assess the potential impact of competing interests. We analysed the association between financial and other competing interests and authors' conclusions in randomised clinical trials published in the $B M J$. We performed the analyses with and without adjustment for potential confounders, including the methodological quality, statistical power, type of experimental intervention, type of control intervention, and specialty.

\section{Methods}

We included all original randomised clinical trials published in the BMJ from 1997 to June 2001. Eligible trials were identified through Medline on PubMed using the key words "bmj" and "random*." We considered trials to be randomised if some form of the word random was used to describe the method of allocation. We excluded articles that referred to previous publications for a description of the study design.

From each trial, we gathered data on authors' conclusions, competing interests, methodological quality, sample size (number of patients per intervention arm), whether the preset sample size was estimated and reached, specialty, type of intervention (pharmacological or non-pharmacological), and type of control (drug, placebo, no intervention, nursing, or surgery).

We defined authors' conclusions as the original investigators' reported interpretation of the extent to which the overall trial results favoured the experimental over the control intervention. We graded authors' conclusions according to phrasing in the abstract and the summarised conclusion on a previously validated 6 point scale ${ }^{2}$ (box). Higher scores indicate more posi-

\author{
Cochrane \\ Hepatobiliary \\ Group, \\ Copenhagen Trial \\ Unit, Centre for \\ Clinical \\ Intervention \\ Research, \\ Copenhagen \\ University Hospital, \\ Department 7102, \\ H:S Rigshospitalet, \\ DK-2100 \\ Copenhagen, \\ Denmark \\ Lise L Kjaergard \\ research fellow \\ Bodil Als-Nielsen \\ research fellow \\ Correspondence to: \\ L L Kjaergard \\ Kjaergard@ctu.rh.dk \\ bmj.com 2002;325:249
}


Scale used to grade authors' conclusions

Experimental intervention highly preferred and should now be considered the standard intervention in all patients or similar (6 points)

Experimental intervention preferred to standard but further trials still indicated; may be more costly or similar disclaimer (5 points)

Experimental and control intervention about equal but experimental intervention successful because of minor advantage (4 points)

Experimental and control intervention about equal, but experimental intervention (3 points) disappointing as control intervention had some minor advantage Control intervention preferred to experimental intervention but experimental intervention might be promising under some circumstances or similar (2 points)

Control intervention highly preferred and is best alternative; should be considered the standard intervention in all patients or similar (1 point)

tive conclusions towards the experimental intervention: scores of 1-3 favoured the control and scores of 4-6 favoured the experimental intervention.

We defined competing interests as anything that may influence professional judgment. We considered funding from for profit organisations-that is, companies that may incur financial gain or loss depending on the outcome-to be financial competing interests. Trials funded by for profit organisations alone and trials funded by both for profit and non-profit organisations were analysed separately. Other competing interests were defined as personal, academic, political, or similar competing interests declared by authors.

We assessed the methodological quality by looking at three components ${ }^{4-6}$ : generation of allocation sequence (adequate, unclear (not reported), or inadequate (quasi-randomised)); concealment of allocation (adequate, unclear (not reported), or inadequate); and blinding (adequate, unclear, or not performed).

One author extracted data unblinded. The other author who was blinded with regard to funding and other competing interests assessed authors' conclusions and methodological quality. The interclass correlation coefficient between blinded and unblinded assessment of authors' conclusions was 0.89 (95\% confidence interval 0.85 to 0.92), indicating good agreement between observers. Consensus was reached in all cases before analyses.

\section{Statistical methods}

We assessed discrepancies between groups of trials by analysis of variance. ${ }^{7}$ Authors' conclusions were log transformed to approximate a normal distribution and to achieve homogeneity of variance. We analysed the association between competing interests and authors' conclusions with and without adjustment for potential confounders - that is, methodological quality, sample size (log transformed), whether the preset sample size was determined and reached, the specialty, type of experimental intervention, and control intervention. We used the step down Bonferroni method to adjust for multiple comparisons by increasing the degrees of freedom. Accordingly, the level of significance was set to $5 \%$. The analyses were performed in SPSS version 10.0 for Windows.

\section{Results}

We identified 684 eligible references of which 159 randomised clinical trials fulfilled our inclusion criteria. In 94 of these trials authors declared that they had no competing interests. In 65 trials authors had competing interests because they had received funding from for profit organisations (27 trials), funding by both for profit and non-profit organisations (19 trials), or other competing interests-for instance, personal, academic, or political (19 trials).

In most trials, authors' conclusions favoured the experimental intervention (table). The generation of the allocation sequence was adequate in 135 trials, unclear in 23 trials, and inadequate in one trial. Concealment of allocation was adequate in 109 trials, unclear in 45 trials, and inadequate in five trials. Forty one trials were double blind, 19 trials were single blind, and 99 trials were not blinded. The mean sample size was 485 (SE 130) patients per intervention arm. Preset sample size calculations were reported in 123 trials and were reached in 103 trials. The trials were in psychiatry, palliative care/nursing, infections, cardiology, gastroenterology, gynaecology/obstetrics, stroke, pain, allergy, obstructive lung diseases, alcohol/drug abuse, and orthopaedic surgery. The experimental intervention was pharmacological in 99 trials and nonpharmacological in 60 trials. The control intervention was nursing in 29 trials, drugs in 27 trials, surgery in 5 trials, placebo in 31 trials, and nothing in 67 trials.

The unadjusted analyses showed a significant association between competing interests and authors' conclusions $\left(r^{2}=0.10 ; \mathrm{P}=0.001\right)$. Authors' conclusions were not significantly different in trials without competing interests, trials funded by both for profit and non-profit organisations, and trials with other competing interests. As shown in the figure, authors' conclusions in trials funded by for profit organisations alone significantly favoured experimental interventions compared with trials without competing interests (mean difference 0.48 (SE 0.13), $\mathrm{P}=0.014$ ), trials funded by both for profit and non-profit organisations $(0.30$ (SE 0.10), $\mathrm{P}=0.003)$, and trials with other competing interests (0.45 (SE 0.13), $\mathrm{P}=0.006$ ). Adjustment for potential confounders did not affect the association between competing interests and authors' conclusions $\left(r^{2}=0.11 ; \mathrm{P}=0.002\right)$. None of the extracted confounders was significantly associated with authors' conclusions.

Authors conclusions in 159 randomised clinical trials published from January 1997 to June 2001 in the BMJ

\begin{tabular}{lc} 
Score for conclusions & No \\
\hline 1 & $10)$ of trials \\
\hline 2 & $5(3)$ \\
\hline 3 & $33(21)$ \\
\hline 4 & $21(13)$ \\
\hline 5 & $39(25)$ \\
\hline 6 & $51(32)$ \\
\hline
\end{tabular}

*Higher scores favour experimental intervention (see box). 


\section{Discussion}

In a sample of randomised clinical trials published in the $B M J$ from January 1997 to June 2001 we found that authors' conclusions were positively associated with financial competing interests. Other competing interests such as personal or academic were not significantly associated with authors' conclusions. Inadequate methodological quality, greater statistical power, specialty, and type of intervention and control did not explain these findings.

\section{Strengths and weaknesses of study}

The included trials covered 12 specialties. A more homogenous group of trials-for example, trials examining one therapeutic question-would have allowed an analysis of the association between competing interests and the trials' quantitative results. Such an analysis, however, would not capture the overall trade off between the efficacy and safety of the interventions. This trade off, which was summarised in authors' conclusions, is important as it may form the basis for subsequent treatment recommendations.

We included trials published in the $B M J$ after the introduction of the CONSORT statement. ${ }^{8}$ We chose this sample because of the consistent reporting of competing interests in the $B M J$ and to avoid confounding by editorial policies, language bias, and variations in methodological quality. We cannot exclude that some competing interests were not revealed by authors. Furthermore, given the declared editorial policies, ${ }^{1}$ the $B M J$ may be considered one of the leading medical journals with respect to the reporting of competing interests. It is possible that the requirement to disclose competing interests will discourage authors with competing interests from submitting biased trials to the $B M J$. Accordingly, we may have underestimated the general association between competing interests and authors' conclusions. Conversely, apart from random error, we have not been able to identify any reasons why the results of the present study should be false positive.

The $B M J$ publishes fewer pharmacological trials than other general medical journals. ${ }^{9}$ According to our adjusted analyses, the association between financial

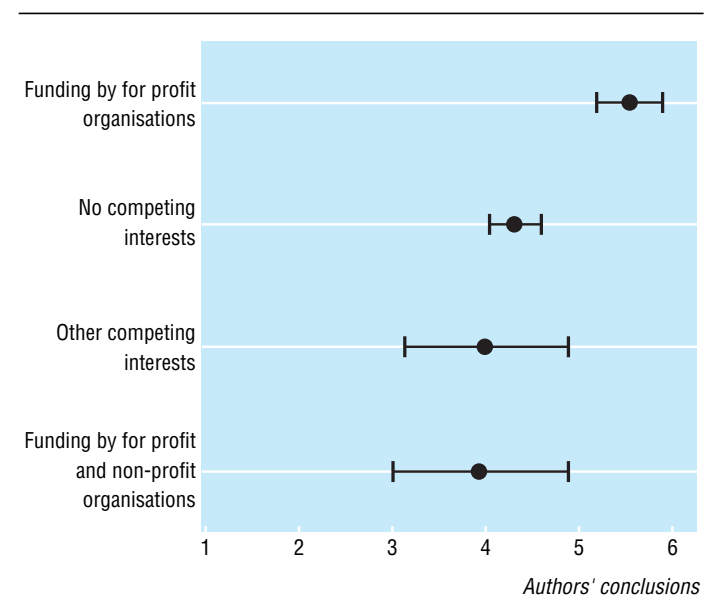

Source of funding and authors' conclusions assessed by 6 point scale (higher scores favour experimental intervention). Points are means with $95 \%$ confidence intervals
What is already known on this topic

Financial competing interests may influence authors' conclusions-for instance, interpretation of whether results favour the experimental or control intervention

Trials of antipsychotic drugs for schizophrenia funded by drug companies were more likely to show a benefit of treatment

It is not known whether other competing interests, such as personal, academic, or political, are associated with authors' conclusions.

\section{What this study adds}

In pharmacological and non-pharmacological randomised clinical trials from 12 specialties financial competing interests were significantly associated with authors' conclusions

The association did not reflect inadequate methodological quality, greater statistical power, or use of inactive control interventions

Personal, academic, and political competing interests were not significantly associated with authors' conclusions.

competing interests and authors' conclusions was significant in pharmacological as well as nonpharmacological trials. Our findings may be relevant only to trials published in the $B M J$, although we cannot identify any reason why this should be the case.

Our results increase the external validity of previous evidence. ${ }^{2}{ }^{3}$ In accordance with Djulbegovic and colleagues, ${ }^{2}$ we found that trials sponsored by for profit organisations significantly more often compared the experimental interventions with placebo or no intervention (data not shown). However, according to our adjusted analyses, this did not explain why authors' conclusions were more favourable towards experimental interventions in trials funded by for profit organisations. Our results also concur with a systematic review by Wahlbeck and colleagues, who showed that the quantitative results of trials were significantly more favourable towards antipsychotic drugs if they were funded by for profit organisations. ${ }^{3}$ The combined evidence supports suggestions that systematic reviews should include sensitivity analyses with regard to funding. ${ }^{3}$

\section{Mechanisms and implications}

Our results cannot show the causes of the association between financial competing interests and authors' conclusions. Profit organisations, by skill or by chance, may fund only those trials in which the experimental intervention is significantly better than the control intervention.

Alternative explanations could be variations in methodological quality or statistical power. According to our adjusted analyses, these aspects could not explain the association between financial competing interests and authors' conclusions. We cannot, however, confirm or refute previous suggestions that the association reflects a violation of equipoise-that is, lack of 
substantial uncertainty about the relative value of one treatment versus another. ${ }^{2}$ Previous evidence indicates that trials sponsored by industry are more likely to be affected by publication bias. ${ }^{10}$ Publication bias could therefore explain the findings of the present study.

Some have argued that industrial funding has undue influence on the research agenda. ${ }^{11}$ Others state that a submitted manuscript should be considered the intellectual property of authors, not the study sponsor. ${ }^{12}$ A reliable assessment of this question depends on the transparency of the reporting. ${ }^{1}$ The CONSORT statement ${ }^{813}$ and similar standardised reporting guidelines could consider the importance of adequate reporting of funding. The reader can make an assessment only if the information is clearly presented.

We thank Christian Gluud for critical revision of an earlier draft of the paper.

Contributors: LLK drafted the paper and performed the literature searches and statistical analyses. Both authors performed data extraction, wrote the paper, and acknowledged the final version. LLK is guarantor.

Funding: Danish Medical Research Council; 1991 Pharmacy Foundation, Denmark; Copenhagen Hospital Corporation Medical Research Council; Danish Institute of Health Technology Assessments.
Competing interests: None declared.

Smith R. Beyond conflict of interest. Transparency is the key. BMJ 1998;317:291-2

2 Djulbegovic B, Lacevic M, Cantor A, Fields KK, Bennett CL, Adams JR, et al. The uncertainty principle and industry-sponsored research. Lancet 2000;356:635-8.

3 Wahlbeck K, Adams C. Beyond conflict of interest. Sponsored drug trials show more favourable outcomes. BMJ 1999;318:465.

4 Schulz KF, Chalmers I, Hayes RJ, Altman DG. Empirical evidence of bias. Dimensions of methodological quality associated with estimates of treatment effects in controlled trials. JAMA 1995;273:408-12.

5 Moher D, Pham B, Jones A, Cook DJ, Jadad AR, Moher M, et al. Does quality of reports of randomised trials affect estimates of intervention efficacy reported in meta-analyses? Lancet 1998;352:609-13.

6 Kjaergard LL, Villumsen J, Gluud C. Reported methodologic quality and discrepancies between large and small randomized trials in metaanalyses. Ann Intern Med 2001;135:982-9.

7 Altman D, Bland JM. Comparing several groups using analysis of variance. $B M J$ 1996;312:1472-3.

8 Begg C, Cho M, Eastwood S, Horton R, Moher D, Olkin I, et al. Improving the quality of reporting of randomized controlled trials. The CONSORT statement. JAMA 1996;276:637-9.

9 Egger M, Bartlett C, Jüni P. Are randomised controlled trials in the BMJ different? [letter]. BMJ 2001;323:1253-4.

10 Easterbrook PJ, Berlin JA, Gopalan R, Matthews DR. Publication bias in clinical research. Lancet 1991;337:867-72.

11 Dieppe P, Chard J, Tallon D, Egger M. Funding clinical research. Lancet 1999;353:1626.

12 Davidoff F, DeAngelis CD, Drazen JM, Hoey J, Højgaard L, Horton R, et al. Sponsorship, authorship, and accountability. Ann Intern Med 2001;135:463-6.

13 Moher D, Schulz K, Altman D. The CONSORT statement: revised recommendations for improving the quality of reports of parallel-group randomised trials. Lancet 2001;357:1191-4.

(Accepted 20 February 2002) 